\title{
Determinação da atenuação de sinais em ambientes indoor via lógica nebulosa
}

\section{Indoor signal attenuation assessment via fuzzy logic}

\author{
Lia Toledo Moreira Mota ${ }^{1}$; Alexandre de Assis Mota ${ }^{2}$; Rina Rondon Gonzalez ${ }^{3}$
}

\section{Resumo}

\begin{abstract}
Este trabalho propõe uma metodologia para a determinação da atenuação do sinal transmitido em ambientes indoor (AI) baseada na aplicação de Lógica Nebulosa (LN). Esse método consiste, basicamente, no emprego de lógica difusa e de um conjunto de regras que permitem a inferência do parâmetro $\beta$ utilizado no Modelo de Propagação do Sinal de Shadowing (MPSS) comumente utilizado na determinação da atenuação de sinais de rádio e bastante difundido na literatura. Os resultados obtidos a partir da aplicação da metodologia proposta mostraram-se bastante satisfatórios, uma vez que foram muito próximos dos obtidos através de métodos experimentais.
\end{abstract}

Palavras-chave: Atenuação de Sinal. Ambientes Indoor. Redes sem Fio. Lógica nebulosa. Modelo de Propagação do Sinal de Shadowing.

\begin{abstract}
This work focuses on the analysis of signal's attenuation in indoor environments using a fuzzy logic approach based on the Shadowing Signal Propagation Model (SSPM). The SSPM allows the characterization of the attenuation caused by the environment through the $\beta$ parameter present in this model. In addition to this, the Fuzzy Logic provides a form of approximate reasoning that allows the treatment of problems with incomplete, vague and imprecise information. Also, it offers a simple way to obtain a possible solution for a problem using the heuristic knowledge about a particular situation. The results show that the methodology produced satisfactory results, close to the ones yielded by experimental methods.

Key words: Signal Attenuation. Indoor. Wireless Networks. Fuzzy Logic. Shadowing Signal Propagation Model.
\end{abstract}

\section{Introdução}

$\mathrm{Na}$ atualidade, a demanda por serviços oferecidos sobre Redes Sem Fio (RSF), tais como comunicação de voz, dados, vídeo e videoconferência vem crescendo significativamente devido à evolução da indústria de Telecomunicações. Nas RSF, que se caracterizam por um meio de transmissão não guiado, o sinal se propaga livremente pelo espaço, podendo sofrer diferentes tipos de interferência, inclusive as causadas pelo meio (TANEMBAUM, 1996), (RAPPAPORT, 2002).

Considerando que a potência transmitida através do link se propaga em diferentes direções, o nível de potência do sinal se reduz conforme a distância

\footnotetext{
1 Professora Pesquisadora do Centro de Ciências Exatas, Ambientais e de Tecnologias da Pontifícia Universidade Católica de Campinas, lia.mota@puc-campinas.edu.br

2 Professor Pesquisador do Centro de Ciências Exatas, Ambientais e de Tecnologias da Pontifícia Universidade Católica de Campinas,amota@puc-campinas.edu.br.

3 Engenheira de Computação, Mestre pelo Programa de Pós-graduação em Engenharia Elétrica da PUC-Campinas; rina@cflex. com.br.
} 
percorrida pelo mesmo aumenta; esta diminuição é influenciada por diversos fatores ambientais e de infra-estrutura como, por exemplo, o layout das edificações, o material empregado nas construções, os elementos construtivos presentes nas edificações, os materiais utilizados em portas e janelas, o layout interno dos edifícios, a quantidade e disposição de divisórias, etc. Assim, torna-se necessário medir a atenuação do sinal causada pela presença de obstáculos para que a RSF possa suportar os serviços para os quais foi inicialmente projetada (RAPPAPORT, 2002).

Nesse contexto, o Modelo de Propagação do Sinal de Shadowing (MPSS), amplamente difundido na literatura, permite calcular a potência do sinal de recepção e, conseqüentemente, a atenuação do sinal transmitido, considerando os obstáculos encontrados durante o seu percurso desde o transmissor até o receptor. Essa atenuação é determinada a partir do cálculo de um parâmetro específico deste modelo (parâmetro $\beta$ ). Entretanto, esse parâmetro representa um tipo de ambiente específico, não permitindo, portanto, a caracterização de condições ou ambientes de forma generalizada.

Além disso, as condições ambientais e de infraestrutura afetam a atenuação do sinal de forma aleatória. Assim, estimar o impacto que cada um desses fatores tem na atenuação do sinal poderia resultar em um processo bastante complexo e, dessa forma, torna-se necessário o desenvolvimento de uma metodologia que permita tratar a influência dos fatores mencionados na determinação da atenuação do sinal de uma forma simples e adequada à representação da realidade.

Nesse sentido, o emprego de Lógica Nebulosa (LN) configura-se como alternativa interessante, uma vez que permite tratar problemas com informações vagas, ambíguas ou imprecisas, de forma simples, através do processamento de uma base de conhecimento e regras condicionais que permitem chegar a uma conclusão aproximada ao conhecimento humano. Dessa maneira, este trabalho propõe a utilização de LN para estimar a atenuação do sinal através da inferência do valor do parâmetro $\beta$ utilizado no MPSS.

\section{Conceitos Principais}

\section{Modelo "Shadowing" de Propagação do Sinal:}

Como citado anteriormente, alguns dos fatores que contribuem na degradação da potência do sinal são dados pelas condições ambientais e de infraestrutura, que devem ser adequadamente incluídos no cálculo da potência de recepção do sinal, através de um modelo de propagação do sinal (MPS) que os levem em consideração. Na literatura atual são apresentados vários MPS, desenvolvidos tanto para ambientes indoor como outdoor, dentre os quais pode-se citar: MPS de Log. Distance, MPS de Shadowing, MPS Okamura-Hata, entre outros, tendo cada um deles certas características que os fazem serem escolhidos para diferentes tipos de aplicações (ANDERSEN et al., 1995) (RAPPAPORT, 2002) (HASHEMI, 1993), (HOLMA; TOSKALA, 2000), (SKLAR, 1997), (SARKAR et al., 2003), (INTERNATIONAL TELECOMMUNICATION UNION, 2008) (YARKONI; BLAUNSTEIN, 2006).

Dentre os modelos mencionados acima, o MPS de Shadowing é recomendado para a planificação de uma RSF para ambientes indoor e se diferencia dos demais porque considera a atenuação sofrida pelo sinal através de seu percurso por diferentes ambientes, cujo valor está relacionado a um parâmetro Beta $(\beta)$, como também, a uma variável aleatória log-normal $\mathrm{X}_{\mathrm{dB}}$ com média zero, que expressa a incerteza do sinal recebido em cada ponto (RAPPAPORT, 2002), (OLIVEIRA, 2008). O MPS de Shadowing é definido conforme a equação (1).

$$
\left[\frac{P_{R}(d)}{P_{R}\left(d_{0}\right)}\right]_{\mathrm{dB}}=-10 \beta \log \left(\frac{d}{d_{0}}\right)+X_{d B}
$$


Nessa equação:

- $\mathrm{P}_{\mathrm{R}}(\mathrm{d})$ representa a potência de recepção a uma distância d;

- $\mathrm{P}_{\mathrm{R}}\left(\mathrm{d}_{0}\right)$ representa a potência de recepção a uma distância d0;

- $\beta$ é o fator de perda de percurso e representa a obstrução ocasionada pelo ambiente;

- $\mathrm{X}_{\mathrm{dB}}$ é uma variável aleatória com distribuição gaussiana de média zero e desvio padrão $\sigma$, os quais são expressados em $\mathrm{dB}$.

Em especial, o parâmetro $\beta$ é utilizado para caracterizar a atenuação em diferentes ambientes. Em Rappaport (2002), é apresentada uma classificação para os valores de $\beta$ em ambientes com obstrução e sem obstrução (ambientes indoor - AI e ambientes outdoor - AO, respectivamente), conforme a Tabela 1 .

Tabela 1. Valores de $\beta$ para AI e AO

\begin{tabular}{ll}
\hline Ambiente & $\boldsymbol{\beta}$ \\
\hline Espaço Livre & 2 \\
Ambiente Urbano & $2,7-3,5$ \\
Ambiente Urbano sombreado & $3-5$ \\
Linha de Visada em Edifícios & $1,6-1,8$ \\
Obstrução em Edifícios & $4-6$ \\
Obstrução em Fábricas & $2-3$ \\
\hline
\end{tabular}

A classificação para ambientes indoor dada na Tabela 1 faz uma diferenciação entre locais com linha de visada para os quais associa $\beta$ a valores entre 1,6 e 1,8; e locais onde existe obstrução à propagação do sinal, podendo $\beta$ assumir um valor entre 2 e 6 dependendo do ambiente (fábrica, edifício ou zona urbana). Contudo, para poder obter um valor de $\beta$ mais próximo da caracterização real do ambiente, é necessário contemplar todas as variáveis que influenciam de forma significativa a atenuação do sinal. Assim, a utilização de modelos matemáticos determinísticos para descrever a influência desses fatores poderia se tornar bastante complexa, devido à natureza estocástica e não-linear das variáveis envolvidas.

\section{Lógica Nebulosa}

A avaliação do impacto relacionado às características ambientais e de infra-estrutura na degradação da potência do sinal torna-se muito importante para que o link possa ser planejado coerentemente. Uma vez que esses fatores são de natureza não determinística, faz-se necessária a utilização de uma ferramenta que viabilize o tratamento deste tipo de variáveis a fim de se determinar, quantitativamente, o grau de atenuação que estas acarretam para o cálculo da potência de recepção.

Nesse contexto, a Lógica Fuzzy (LF) ou Lógica Nebulosa (LN) tem se configurado como uma ferramenta poderosa para resolver problemas complexos onde as informações se apresentam de forma incompleta, vaga, ambígua e imprecisa (CAMARGOS, 2002). Assim, o uso da Lógica Nebulosa ofereceria um mecanismo simples para a perda de percurso, representada pelo parâmetro $\beta$, que qualifica a atenuação gerada pelos diversos obstáculos presentes em um ambiente.

A LN tem sido adotada nas últimas décadas como proposta para tratamento de variáveis que contêm informações vagas, ambíguas e imprecisas. A sua aplicabilidade se estende a diversas áreas tecnológicas, como sistemas de otimização, mecânicos, controladores e sistemas de automação (ZADEH, 1965) (PASSINO; YURKOVICH, 1998) (MOTA et al., 2007), onde o conhecimento inerente à dinâmica da aplicação foi expresso através de um conjunto de base de regras lingüísticas para resolver problemas complexos que contêm variáveis imprecisas. Sua capacidade de representar a imprecisão em conjuntos tratáveis matemática 
e computacionalmente torna a Lógica Nebulosa atrativa para problemas com alto grau de imprecisão quanto ao cenário da aplicação, tal como o problema da determinação dos fatores de atenuação do sinal em diferentes tipos de ambientes.

Sua utilização está baseada na Teoria de Conjuntos Nebulosos, a qual estabelece que para cada elemento que pertence a um conjunto, um grau de pertinência em relação ao conjunto é determinado, sendo que este pode assumir um valor entre 0 e 1 , onde 0 indica pertinência nula ao conjunto, 1 pertinência total e um valor nesse intervalo que indica pertinência parcial. A Figura 1 apresenta o modelo genérico de um controlador nebuloso.

Figura 1. Modelo Genérico de um Controlador Nebuloso.

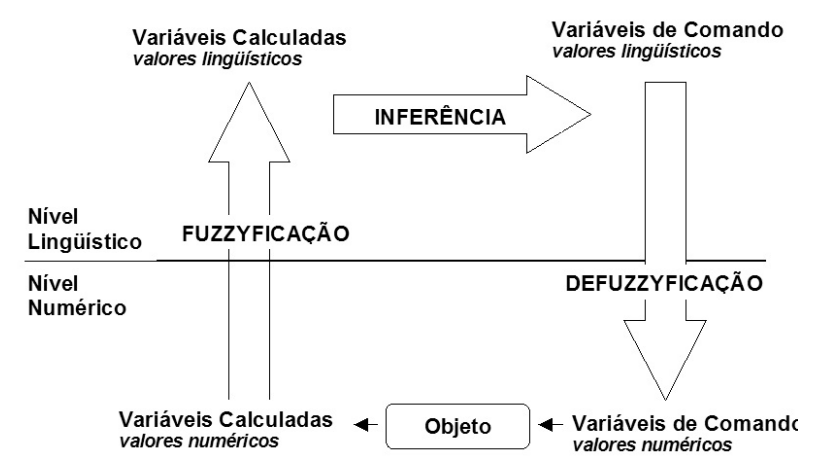

A aplicação da LN se inicia com o processo de fuzzyficação, onde valores numéricos são traduzidos em variáveis lingüísticas. Para isto, são utilizadas funções de pertinência as quais podem ser triangulares, trapezoidais, gaussianas, entre outras. As funções triangulares são particularmente simples e permitem descrever uma relação de pertinência a um conjunto difuso, especialmente quando os elementos do conjunto são discretos (COX, 1999) (TSOULAKAS, 1997).

A Inferência está baseada no conjunto de regras, dado que através da sua aplicação pode ser mapeada a relação entre as variáveis lingüísticas de entrada e saída. Depois do mapeamento, tem lugar o processo de implicação, o qual consiste, basicamente, na ativação de uma regra do tipo "SE condição - ENTÃO conseqüente" para o qual se utilizam as operações de implicação nebulosas. Entre as operações de implicação existentes, podese destacar a implicação Max-Min onde "Max" é dada pela operação "OU" e "Min" pela operação "E".

A defuzzyficação transforma o resultado do processo de inferência em entradas para o sistema, ou seja, traduz os valores lingüísticos em numéricos. Entre os métodos mais utilizados podese destacar o Método do Centro de Gravidade que determina como saída o centro de gravidade da área de cada conjunto fuzzy implicado. Outro método amplamente difundido na literatura é o Método da Média dos Máximos que retorna a média dos pontos que possuem maior grau de pertinência. As expressões que representam cada um desses métodos encontram-se apresentadas, respectivamente, pelas equações (2) e (3).

$$
\begin{aligned}
& u=\frac{\sum_{i=1}^{n} x_{i} \cdot A\left(x_{i}\right)}{\sum_{i=1}^{n} A\left(x_{i}\right)} \\
& u^{*}=\sum_{m=1}^{M}=\frac{u_{m}}{M}
\end{aligned}
$$

Onde, na equação (2), n é o número de pontos envolvidos com a operação de defuzzyficação; $\mathrm{x}_{\mathrm{i}}$ é o valor da variável de entrada correspondente ao ponto i; $\mathrm{A}\left(\mathrm{x}_{\mathrm{i}}\right)$ é o valor da função de pertinência correspondente ao valor de entrada $\mathrm{x}_{\mathrm{i}}$; e, na equação (3), $\mathrm{M}$ é o número de pontos envolvidos com a operação de defuzzyficação; e $\mathrm{u}_{\mathrm{m}}$ é o valor da função de pertinência dada pelo valor de entrada correspondente ao ponto $\mathrm{m}$.

Neste trabalho, a implementação da LN será desenvolvida usando o modelo de controlador fuzzy descrito em Passino e Yurkovich (1998). Assim, as funções de pertinência serão modeladas como as de 
forma triangular, uma vez que os dados de entrada apresentam um comportamento discreto e cada um destes ocasiona um diferente impacto na variável de saída. Do mesmo modo, para a realização do processo de Inferência vai ser usado o método de Mandani, min (.), uma vez que, de acordo com Passino e Yurkovich (1998) é de simples utilização.

\section{Metodologia}

Com o propósito de validar o modelo nebuloso proposto neste trabalho, os resultados obtidos após a etapa de defuzzificação foram confrontados com resultados empíricos obtidos em um estudo de propagação realizado nas salas de aula da Pontifícia Universidade Católica de Campinas descrito em (BRANQUINHO; REGGIANI; ANDREOLLO, 2005), caracterizando um ambiente indoor com 6 salas, dispostas conforme indicado na Figura 2.

Neste ambiente, as paredes externas e a parede central, que divide as salas 3 e 4, são de alvenaria. As outras paredes são formadas por divisórias convencionais. No Access Point (AP) foi utilizada uma antena setorial de noventa graus. Para a coleta de dados foi utilizado um notebook equipado com uma placa de rede sem fio e com uma antena monopolo vertical. Durante o estudo, ambos foram posicionados a 1,85 metros do chão, permitindo espaçamento entre os pontos de medida, em média, de $30 \mathrm{~cm}$.

Figura 2. Ambiente Indoor Estudado.

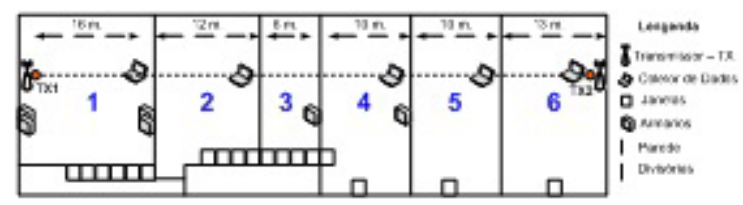

\section{Caracterização das salas do ambiente de testes}

Como já descrito, o ambiente indoor selecionado para o teste da metodologia proposta neste trabalho é composto por seis salas de aula da PUC-Campinas, caracterizadas pela presença dos seguintes objetos:

- Sala 1: Dentro dessa sala (onde foi posicionado o AP transmissor TX1) existem duas paredes de alvenaria, janelas de vidro fino e foi identificada uma forte presença de armários metálicos.

- Sala 2: Possui uma parede de alvenaria e uma divisória de madeira fina. Também foi identificada a presença de janelas.

- Sala 3: Possui uma parede de alvenaria, uma divisória de madeira e janelas. Deve-se salientar que o tamanho desta sala é muito menor quando comparado com o tamanho das duas primeiras.

- Sala 4: Esta sala apresenta uma característica em especial, uma vez que a divisória dessa sala com a sala 3 que é feita de alvenaria e não de madeira. Também possui outras duas paredes de alvenaria.

- Sala 5: Esta sala se caracteriza por ser pequena em comparação as restantes e tem 2 paredes de alvenaria e 2 divisórias de madeira.

- Sala 6: Esta sala é a última do ambiente em estudo (onde foi posicionado o AP de recepção TX2). Também é pequena, se comparada com as demais, e possui 2 divisórias de madeira e 2 paredes de alvenaria.

A Tabela 2 apresenta de maneira resumida a caracterização de cada uma das salas do ambiente estudado.

Tabela 2. Caracterização individual de cada sala.

\begin{tabular}{|l|c|c|c|c|c|c|}
\cline { 2 - 8 } \multicolumn{1}{c|}{} & \multicolumn{7}{c|}{ POR SALA } \\
\hline \multicolumn{1}{c|}{ Característica } & Sala 1 & Sala 2 & Sala 3 & Sala 4 & Sala 5 & Sala 6 \\
\hline Paredes & 1 & 1 & 1 & 3 & 2 & 2 \\
\hline Divisórias & 0 & 1 & 1 & 0 & 1 & 1 \\
\hline Janelas & 7 & 4 & 4 & 2 & 1 & 1 \\
\hline Armários Metálicos & 4 & 0 & 1 & 1 & 0 & 0 \\
\hline $\begin{array}{l}\text { Comprimento das } \\
\text { salas (metros) }\end{array}$ & 16 & 12 & 6 & 10 & 10 & 13 \\
\hline $\begin{array}{l}\text { Distância acumulada } \\
\text { (metros) }\end{array}$ & 16 & 28 & 34 & 44 & 54 & 67 \\
\hline
\end{tabular}

Conforme exposto anteriormente, a potência da recepção varia em função da distância e obstrução ocasionada pelo meio. Assim, para o estudo de propagação proposto, a distância considerada em 
cada sala deve ser composta pela somatória dos tamanhos das salas que a antecedem, caracterizando assim, uma distância acumulada. De forma análoga, no caso dos objetos que obstruem o sinal, também deve ser considerada a composição total das salas que antecedem aquela onde será estimado o parâmetro $\beta$. A Tabela 3 mostra o resumo da caracterização acumulada das salas para o ambiente estudado.

Tabela 3. Caracterização acumulada em cada sala.

\begin{tabular}{|l|c|c|c|c|c|c|}
\cline { 2 - 8 } & \multicolumn{6}{|c|}{ ACUMULADOS POR SALA } \\
\hline Caracteristica & Sila 1 & Sala 2 & Sala 3 & Sala 4 & Sala 5 & Sala 6 \\
\hline Paredes & 1 & 2 & 3 & 6 & 8 & 10 \\
\hline Divisárias & 0 & 1 & 2 & 2 & 3 & 4 \\
\hline Janelas & 7 & 11 & 15 & 17 & 18 & 19 \\
\hline $\begin{array}{l}\text { Armaíries Metílicos } \\
\text { Tamanho das salas } \\
\text { (metres) }\end{array}$ & 4 & 4 & 5 & 6 & 6 & 6 \\
\hline $\begin{array}{l}\text { Distância acumulada } \\
\text { (metres) }\end{array}$ & 16 & 12 & 6 & 10 & 10 & 13 \\
\hline
\end{tabular}

\section{Variáveis Nebulosas de Entrada}

As variáveis nebulosas de entrada para o sistema nebuloso representam os dados que impactam na propagação do sinal no ambiente indoor e foram implementadas de forma a representar as características de cada sala, descrita anteriormente. As variáveis implementadas foram:
a. Paredes de Alvenaria- (PAR)
b. Divisórias de Madeira - (DIV)
c. Janelas - (JAN)
d. Armários Metálicos - (AM)
e. Distância - (DIS)
f. Tamanho - (TAM)

Os valores nebulosos para cada uma dessas variáveis foram determinados de acordo com os resultados do estudo de propagação no ambiente de testes, descritos em Branquinho, Reggiani e Andreollo (2005). Em relação às funções de pertinência selecionadas para a qualificação de todas as variáveis nebulosas, adotou-se a função triangular, pois, por um lado, segundo os resultados experimentais, os valores obtidos são bastante discretos, e por outro, essa função requer baixo esforço computacional e conduz a resultados satisfatórios. A Figura 3 apresenta esse tipo de função de pertinência, onde o eixo x representa os valores da variável de entrada, o eixo $\mu$ representa os valores da pertinência à função, a é o limitante inferior da função, b é o valor modal (onde $\mu=1$ ) e c é o limitante superior da função.

Figura 3. Função de pertinência triangular.

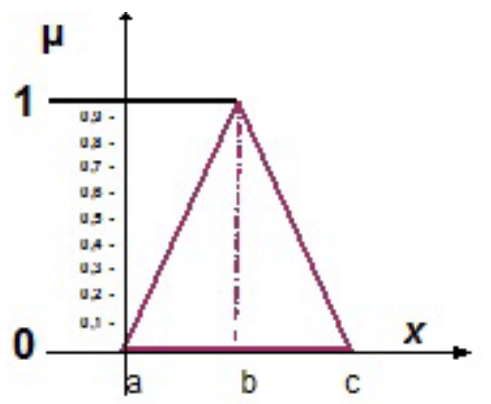

A Tabela 4 apresenta os valores inferior, modal e superior para os conjuntos nebulosos que caracterizam as variáveis de entrada do sistema nebuloso.

Tabela 4. Caracterização das variáveis nebulosas de entrada.

\begin{tabular}{|c|c|c|c|}
\hline Variável Nebulosa & $\begin{array}{c}\text { Valor Linguístico } \\
\text { associado (conjunto } \\
\text { nebuloso) }\end{array}$ & Tipo de função & $\begin{array}{c}\text { Valores inferior, } \\
\text { modal e superior do } \\
\text { conjunto }\end{array}$ \\
\hline \multirow{3}{*}{ PAR } & Baixo & triangular & $(0,1,4)$ \\
\cline { 2 - 4 } & Médio & triangular & $(2,6,8)$ \\
\cline { 2 - 4 } & Alto & triangular & $(7,9,11)$ \\
\hline \multirow{2}{*}{ DIV } & Poucas & triangular & $(-1,0,2)$ \\
\cline { 2 - 4 } & Muitas & triangular & $(1,3,5)$ \\
\hline \multirow{3}{*}{ JAN } & Baixo & triangular & $(0,4,9)$ \\
\cline { 2 - 4 } & Médio & triangular & $(8,11,16)$ \\
\cline { 2 - 4 } & Alto & triangular & $(14,19,22)$ \\
\hline \multirow{3}{*}{ AM } & Baixo & triangular & $(0,1,2)$ \\
\cline { 2 - 4 } & Médio & triangular & $(1,3,5)$ \\
\cline { 2 - 4 } & Alto & triangular & $(4,6,8)$ \\
\hline \multirow{2}{*}{ DIS } & Baixa & triangular & $(5,20,39)$ \\
\cline { 2 - 4 } & Média & triangular & $(35,48,52)$ \\
\cline { 2 - 4 } & Alta & triangular & $(50,59,70)$ \\
\hline \multirow{2}{*}{ TAM } & Pequeno & triangular & $(6,10,14)$ \\
\cline { 2 - 4 } & Grande & triangular & $(12,15,20)$ \\
\hline
\end{tabular}

Inferência e variável nebulosa de saída:

Esta etapa envolve a ativação da base de regras que permitirá mapear a relação entre os valores obtidos na etapa de fuzzyficação e os valores da variável nebulosa de saída, através de um mecanismo específico de inferência, ou, em outras 
palavras, através de uma relação de implicação. Considerando que o objetivo proposta neste trabalho é calcular o grau de obstrução representado pelo parâmetro $\beta$, a variável de saída mais adequada para a representação desse propósito é a obstrução OBS, descrita conforme segue, pela Tabela 5 e pela Figura 4.

Tabela 5. Caracterização das variáveis nebulosas de entrada.

\begin{tabular}{|c|c|c|c|}
\hline Variável Nebulexa & $\begin{array}{l}\text { Valor Lingivistico } \\
\text { nossociado (conjunto } \\
\text { nebulaso) }\end{array}$ & Tipo de funçầ & $\begin{array}{c}\text { Valores inferior, } \\
\text { modal e superior do } \\
\text { conjunto }\end{array}$ \\
\hline \multirow{6}{*}{ Olts } & Nula & triangular & $(-25,-1.8 .0001)$ \\
\hline & Muito Elaiso & trisangular & $(0,1,7,2.4)$ \\
\hline & Baixa & triangular & (2.3.3.5) \\
\hline & Medio & triangular & $(3.2,4,4.7)$ \\
\hline & Alio & triangular & $(4,3,5,3,6,3)$ \\
\hline & Muito Alta & triangular & $(6.2,8,10)$ \\
\hline
\end{tabular}

Figura 4. Conjuntos da variável de saída Obstrução (OBS).

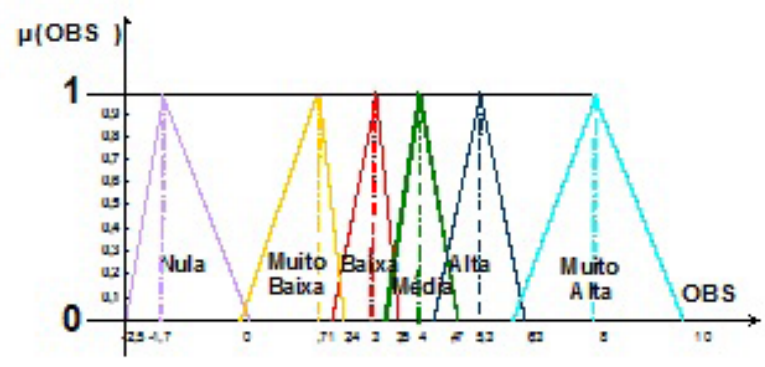

Assim, o mecanismo de inferência está fundamentado na relação entre as funções de pertinência da variável de saída OBS e os resultados provenientes da etapa de fuzzyficação, por meio de uma base de regras nebulosas que viabilizam essa inferência. Neste trabalho, foram implementadas 103 regras; uma delas está apresentada a seguir, a título de exemplificação:

SE (PA é Baixo) E (DIV é Poucas) E (JAN é Baixa) E (DIS é Baixa) E (TAM é Grande) E (AM é Médio) ENTÃO (OBS é MUITO ALTA)

Para a aplicação efetiva da base de regras, é necessário adotar uma relação de implicação, que estipula a pertinência de uma determinada situação aos qualificativos da variável nebulosa de saída com base nos resultados obtidos para as pertinências das variáveis de entrada. Para tanto, existem diversas operações de implicação; neste trabalho, com já citado, foi escolhida a de Mandani, min (.), devido à sua simplicidade de uso.

\section{O Sistema de Regras Nebulosas}

Como exposto anteriormente, um sistema fuzzy tem principalmente três etapas: fuzzyficação, inferência e defuzzyficação, em cada uma das quais se realizam determinados processos. O fluxograma apresentado na Figura 5 apresenta a metodologia proposta neste trabalho. Nesse diagrama estão explicitados os processos e sub-processos que cada uma destas etapas principais envolve, assim como outras atividades de entrada do processo.

Figura 5. Fluxograma da Metodologia Proposta.

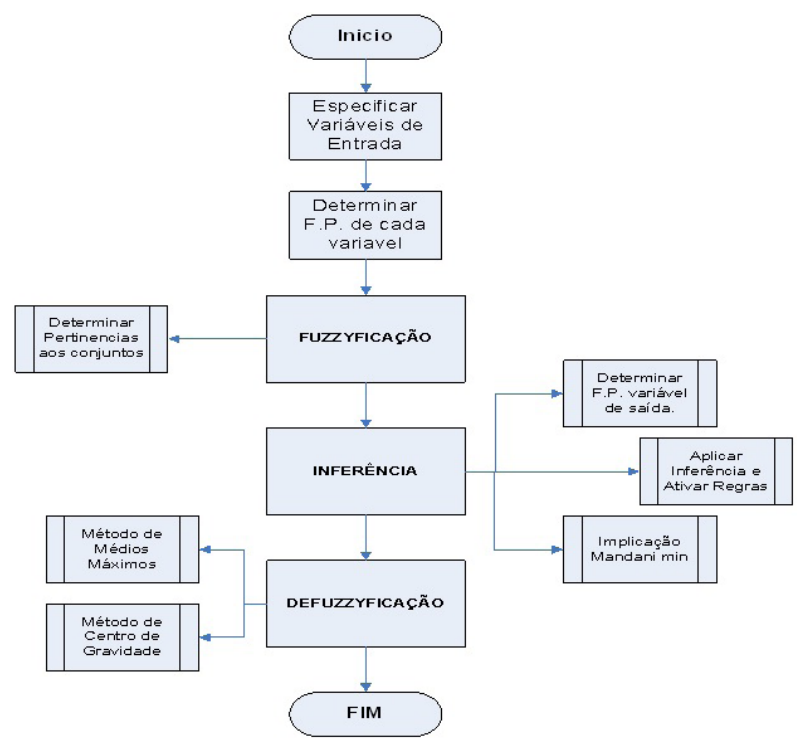

\section{Etapa de Defuzzyficação}

O propósito desta etapa é o de transformar um dado nebuloso em um dado quantitativo, ou "crisp". Neste trabalho, esta etapa foi implementada aplicando os métodos de Centro de Gravidade 
e Médios Máximos, a partir de cálculo manual (para aplicação direta) e computacional, a partir de aplicativo desenvolvido na plataforma Java. Tanto para o método do Centro de Gravidade, quanto para o método dos Médios Máximos, foram escolhidos 5 pontos pertencentes à área de pertinência para a execução do calculo manual. Uma vez que estes são pontos de referência, incide-se em erro inerente ao conjunto selecionado na determinação da solução, ou seja, na medida em que um maior número de pontos é avaliado, o resultado é composto por um grau maior de mais discretização, o que conduz a uma menor margem de erro. Os resultados obtidos através de cálculo manual com cinco pontos foram também comparados com os resultados fornecidos pela ferramenta computacional desenvolvida para o tratamento de Sistemas de Lógica Nebulosa, que emprega um total de 1000 pontos para a defuzzyficação.

\section{Resultados}

Os dados pertinentes a cada sala, apresentados na tabela 3 , foram aplicados à metodologia de inferência nebulosa proposta, apresentada na Figura 5. Em seguida, foram comparados com os dados obtidos através de cálculos empíricos, extraídos da referência (BRANQUINHO; REGGIANI; ANDREOLLO, 2005). Para essa comparação, foram adotados os valores defuzzyficados de acordo com o método do centro de gravidade (CG) e média dos máximos (MM), empregando cálculo manual (com 5 pontos) e computacional (com 1000 pontos). A Tabela 6 apresenta a síntese dos resultados obtidos.

Tabela 6. Síntese dos resultados obtidos para as salas de 1 a 6.

\begin{tabular}{|c|c|c|c|c|c|}
\hline \multirow{2}{*}{ Sala } & \multirow{2}{*}{$\begin{array}{c}\text { Calculo } \\
\text { Empirico }\end{array}$} & \multicolumn{2}{|c|}{ Manual } & \multicolumn{2}{c|}{ Computacional } \\
\cline { 3 - 6 } & & $\mathbf{C G}$ & MM & $\mathbf{C G}$ & MM \\
\hline $\mathbf{1}$ & 8.1116 & 8,12 & 8,12 & 8,05 & 7.94 \\
\hline $\mathbf{2}$ & 0,02011 & 1,39 & 1,39 & $-0,14$ & 0,07 \\
\hline $\mathbf{3}$ & $-1,4018$ & $-1,33$ & $-1,34$ & $-0,26$ & $-0,26$ \\
\hline $\mathbf{4}$ & 4.7992 & 5,3 & 5,3 & 5,31 & 5.2 \\
\hline $\mathbf{5}$ & 2.9495 & 2.82 & 2.82 & 1.92 & 2.13 \\
\hline $\mathbf{6}$ & 1,8988 & 2.23 & 2.12 & 1.79 & 2.13 \\
\hline
\end{tabular}

\section{Análise de Resultados - Sala 1}

Como mostrado na Tabela 6, os resultados para a Sala 1 foram muito semelhantes nos diferentes tipos de cálculos. Os erros apresentados entre o cálculo empírico e o manual foram de $0,1 \%$ para os métodos CG e MM; e, em relação ao cálculo computacional, foram de $0,7 \%$ para o método $C G$ e de $2 \%$ para o método MM.

\section{Análise de Resultados - Sala 2}

No caso da Sala 2, os resultados do calculo empírico como do computacional estão próximos; apresentam uma diferença de $85 \%$ para o método CG e $71 \%$ para o método MM, mas o porte dessas porcentagens de erro se justifica pelo baixo valor apresentado pelo método empírico (muito próximo de zero) o que gera distorções quando se analisa o erro percentual. Nesse caso específico, o erro absoluto (0,16 para o CG e 0,05 para o MM) se configura como um indicador de proximidade melhor que o erro percentual. Já a significativa diferença obtida entre o dado empírico e o cálculo manual pode ser justificada em função da escolha dos pontos tomados na defuzzyficação, que podem não ser os mais representativos da variável de saída em relação à sua função de pertinência.

\section{Análise de Resultados - Sala 3}

Neste caso, os métodos manuais e o empírico forneceram resultados bastante similares; um ponto interessante a se destacar na Sala 3 é que os resultados indicam um ganho ao invés de uma perda de sinal. Pode-se observar também que o cálculo computacional mostra, de forma similar, uma tendência para a obtenção de ganho (o sinal negativo, neste caso, indica ganho), embora não na mesma intensidade dos primeiros métodos mencionados. Este é um fato interessante, uma vez que a base de regras foi projetada apenas com base nas situações de atenuação (que representam somente perdas no sinal), mas levando em conta conhecimento 
especialista; o aparecimento de situações onde o sinal da atenuação é invertido, significando ganho, demonstra a capacidade da base de regras de extrapolar o conhecimento contido originalmente nas mesmas para atender a situações que não estão explicitamente descritas na sua geração.

\section{Análise de Resultados - Sala 4}

Os resultados para a Sala 4 podem ser considerados próximos para os diferentes tipos de cálculos. Os erros apresentados entre o cálculo empírico e o manual foram de $10 \%$ para os métodos CG e MM; e, em relação ao cálculo computacional, foram de $11 \%$ para o método CG e de $8 \%$ para o método MM.

\section{Análise de Resultados - Sala 5}

No caso da sala 5, os erros apresentados entre o cálculo empírico e o manual foram de 4\% para os métodos CG e MM; e, em relação ao cálculo computacional, foram de 34\% para o método CG e de $27 \%$ para o método MM. Tendo-se em vista a imprecisão inerente à lógica nebulosa, estes resultados podem ser considerados satisfatórios.

\section{Análise de Resultados - Sala 6}

Assim como no caso da Sala 4, os resultados para a Sala 6 também podem ser considerados próximos para os diferentes tipos de cálculos. Os erros apresentados entre o cálculo empírico e o manual foram de $15 \%$ para o método $\mathrm{CG}$ e de 11\% para o método MM; e, em relação ao cálculo computacional, foram de 5\% para o método $\mathrm{CG}$ e de $11 \%$ para o método MM.

\section{Conclusões}

$\mathrm{Na}$ literatura atual, existem diversos modelos de propagação do sinal que permitem predizer a potência do sinal de recepção. Alguns deles levam em conta os obstáculos encontrados no meio, outros só consideram a distância como fator que ocasiona atenuação do sinal. No entanto, para realizar um cálculo real da potência do sinal de recepção, é necessário levar em conta os obstáculos presentes no meio, já que os mesmos, como salientado neste trabalho, provocam obstrução na transmissão do sinal gerando atenuação da mesma.

Em relação a redes sem fio, por fazerem uso de um meio de transmissão não guiado, deve-se considerar que estão expostas a interferências ocasionadas pela topologia das estruturas e objetos presentes no meio, tais como: paredes, janelas, objetos metálicos, layout de edificações, portas, etc., cujos efeitos na atenuação do sinal são complexos de estimar. Por outro lado, a maioria dos modelos matemáticos, adotados atualmente para tratar desse problema, considera as paredes e andares como fatores que atenuam o sinal, mas não levam explicitamente em conta a quantidade e a natureza dos componentes internos e de layout das edificações, como os objetos metálicos, as janelas, as portas, dentre outros.

Dentre os modelos matemáticos para estimação da atenuação na propagação do sinal, o modelo de Shadowing faz uma caracterização do ambiente através do exponente de perda do percurso Beta, o qual, segundo Rapapport (2002), pode ser um valor arbitrário entre 3 e 6 quando o ambiente é obstruído. A classificação dada pelo citado autor é genérica e não permite contabilizar os elementos que se encontram dentro do ambiente. Pode-se, nesse sentido, afirmar que uma contribuição da modelagem com a base de regras nebulosa proposta nesse trabalho é justamente a de considerar tais elementos.

O estudo realizado demonstrou que, através da aplicação da Lógica Nebulosa para o estudo de atenuação do sinal nos ambientes indoor, torna-se possível a obtenção de valores bastante aproximados aos medidos empiricamente. Nesse sentido, a adoção de Lógica Nebulosa permite o tratamento de dados ambíguos, imprecisos, com presença de um 
certo grau de incerteza. Também permite a solução de problemas onde a modelagem matemática seria complexa. O método de (Max, Min), escolhido pela simplicidade da sua aplicação, foi capaz de fornecer resultados satisfatórios, suficientemente aproximados dos experimentais, verificados para dois métodos de defuzzyficação: os métodos de Centro de Gravidade e Médios Máximos.

Os dados coletados ao longo do trabalho foram comparados com os obtidos através de um sistema computacional de Lógica Nebulosa, implementado na plataforma Java, fornecendo resultados aproximados aos cálculos manuais e aos medidos.

Finalmente, pode-se afirmar que a Lógica Nebulosa demonstrou ser uma possível ferramenta para o tratamento dos fatores de atenuação do sinal, simplificando o processo de caracterização da obstrução do ambiente e permitindo obter resultados aproximados aos das medições reais. Além disso, pôde-se também observar que a base de regras proporcionou um certo grau de inteligência computacional à solução para o problema ao extrapolar o conhecimento contido originalmente na sua geração para a previsão de situações onde ocorreu ganho (e não perda) na intensidade do sinal.

\section{Referências}

ANDERSEN, J. B.; RAPPAPORT, T. S.; YOSHIDA, $\mathrm{S}$. Propagation measurements and models for wireless communications channels. IEEE Communications Magazine, New York, v. 33, n. 1, p. 42-49, 1995.

BRANQUINHO, O.; REGGIANI, N.; ANDREOLLO, A. Modelo de propagação shadowing modificado para ambiente interno para redes PWLAN em 2,4GHz. SIMPÓSIOBRASILEIRODETELECOMUNICAÇÕES, 22., 2005, Campinas. Anais... Campinas: SBrT, 2005

CAMARGOS, F. Lógica nebulosa: uma abordagem filosófica e aplicada. Florianópolis: Departamento de Informática e Estatística /UFSC, 2002.

COX, E. The fuzzy systems handbook. New York: AP Professional, 1999.

HASHEMI, H. The indoor propagation channel. IEEE, New York, v. 81, n. 7, Jul. 1993.
HOLMA, H.; TOSKALA, A. WCDMA for UMTS: radio access for third generation mobile communications. Hoboken: John Wiley, 2000.

INTERNATIONAL

TELECOMMUNICATION

UNION. ITU-R P.1238: propagation data and prediction models for the planning of indoor radiocommunication systems and radio local area networks in the frequency range $900 \mathrm{MHz}$ to $100 \mathrm{GHz}$. Geneva: ITU-R, 2008.

MOTA, L. T. M.; MOTA, A. A.; MORELATO, A. Load behaviour prediction under blackout conditions using a fuzzy expert system. IET Proceedings on Generation, Transmission \& Distribution, Stevenage, v. 1, n. 3, p. 379-387, May 2007.

OLIVEIRA, K. S. L. Avaliação da instabilidade da modulação em diferentes ambientes. 2008. Dissertação (Mestrado em Engenharia Elétrica) - Programa de PósGraduação Stricto-Sensu - Pontificia Universidade Católica de Campinas, Campinas.

PASSINO, K; YURKOVICH, S. Fuzzy control. Menlo park: Addison Wesley Longman, 1998.

RAPPAPORT, T. S. Wireless communications-principles and practice. Upper Saddle River: Prentice Hall, 2002.

SARKAR, T. K.; ZHONG, J.; KYUNGJUNG, K.; MEDOURI, A.; SALAZAR-PALMA, M. A survey of various propagation models for mobile communication. IEEE antennas and propagation magazine, New York, v. 45, n. 3, Jun. 2003.

SKLAR, S. Rayleigh fading channels in mobile digital communication system: part I: characterization. IEEE Communications Magazine, New York, p. 90-100, Jul. 1997.

TANENBAUM, A. Computer networks. 3. ed. New Jersey:Prentice-Hall, 1996.

TSOUKALAS, L.; UHRIG, R. Fuzzy and neural approaches in engineering. New York: Wiley Interscience, 1997.

YARKONI, N.; BLAUNSTEIN, N. Prediction of propagation characteristics in indoor radio communication environments. Progress In Electromagnetics Research, Kuala Lumpur, v. 59, p. 151-174, 2006.

ZADEH, L. Fuzzy sets. Information and control, New York, v. 8, n. 3, p. 338-353, Jun. 1965.

\section{Apêndice: Base de Regras}

(1) SE (PA é Baixo) E (DIV é Pouca) E (JAN é Baixa) E (DIS é Baixa) E (TAM é Grande) E (AM é Alto) ENTÃO (OBS é MUITO ALTA) 
(2) SE (PA é Baixo) E (DIV é Pouca) E (JAN é Média E (DIS é Baixa) E (TAM é Pequeno) E (AM é Médio) ENTÃO (OBS é MUITO BAIXA)

(3) SE (PA é Médio) E (DIV é Pouca) E (JAN é Média) E (DIS é Baixa) E (TAM é Pequeno) E (AM é Médio) ENTÃO (OBS é NULA)

(4) SE (PA é Baixo) E (DIV é Pouca) E (JAN é Média) E (DIS é Baixa) E (TAM é Pequeno) E (AM é Alto) ENTÃO (OBS é NULA)

(5) SE (PA é Médio) E (DIV é Pouca) E (JAN é Média) E (DIS é Baixa) E (TAM é Pequeno) E (AM é Alto) ENTÃO (OBS é NULA)

(6) SE (PA é Baixo) E (DIV é Pouca) E (JAN é Média) E (DIS é Baixa) E (TAM é Grande) E (AM é Média) ENTÃO (OBS é NULA)

(7) SE (PA é Médio) E (DIV é Pouca) E (JAN é Média) E (DIS é Baixa) E (TAM é Grande) E (AM é Média) ENTÃO (OBS é NULA)

(8) SE (PA é Baixo) E (DIV é Pouca) E (JAN é Média) E (DIS é Baixa) E (TAM é Grande) E (AM é Alto) ENTÃO (OBS é NULA)

(9) SE (PA é Média) E (DIV é Pouca) E (JAN é Média) E (DIS é Baixa) E (TAM é Grande) E (AM é Alto) ENTÃO (OBS é NULA)

(10) SE (PA é Baixa) E (DIV é Pouca) E (JAN é Média) E (DIS é Baixa) E (TAM é Pequeno) E (AM é Média) ENTÃO (OBS é NULA)

(11) SE (PA é Baixa) E (DIV é Muitas) E (JAN é Média) E (DIS é Baixa) E (TAM é Pequeno) E (AM é Média) ENTÃO (OBS é NULA)

(12) SE (PA é Baixa) E (DIV é Muitas) E (JAN é Média) E (DIS é Baixa) E (TAM é Pequeno) E (AM é Alto) ENTÃO (OBS é NULA)

(13) SE (PA é Média) E (DIV é Muitas) E (JAN é Média) E (DIS é Baixa) E (TAM é Pequeno) E (AM é Alto) ENTÃO (OBS é NULA)

(14) SE (PA é Baixa) E (DIV é Muitas) E (JAN é Média) E (DIS é Baixa) E (TAM é Grande) E (AM é Média) ENTÃO (OBS é NULA)

(15) SE (PA é Média) E (DIV é Muitas) E (JAN é Média) E (DIS é Baixa) E (TAM é Grande) E (AM é Média) ENTÃO (OBS é NULA)

(16) SE (PA é Baixa) E (DIV é Muitas) E (JAN é Média) E (DIS é Baixa) E (TAM é Grande) E (AM é Alto) ENTÃO (OBS é NULA)

(17) SE (PA é Média) E (DIV é Muitas) E (JAN é Média) E (DIS é Baixa) E (TAM é Grande) E (AM é
Alto) ENTÃO (OBS é NULA)

(18) SE (PA é Baixa) E (DIV é Poucas) E (JAN é Média) E (DIS é Baixa) E (TAM é Pequeno) E (AM é Médio) ENTÃO (OBS é NULA)

(19) SE (PA é Média) E (DIV é Poucas) E (JAN é Média) E (DIS é Baixa) E (TAM é Pequeno) E (AM é Médio) ENTÃO (OBS é NULA)

(20) SE (PA é Baixa) E (DIV é Poucas) E (JAN é Alta) E (DIS é Baixa) E (TAM é Pequeno) E (AM é Médio) ENTÃO (OBS é NULA)

(21) SE (PA é Média) E (DIV é Poucas) E (JAN é Alta) E (DIS é Baixa) E (TAM é Pequeno) E (AM é Médio) ENTÃO (OBS é NULA)

(22) SE (PA é Baixa) E (DIV é Poucas) E (JAN é Média) E (DIS é Baixa) E (TAM é Pequeno) E (AM é Alto) ENTÃO (OBS é NULA)

(23) SE (PA é Média) E (DIV é Muitas) E (JAN é Média) E (DIS é Baixa) E (TAM é Pequeno) E (AM é Alto) ENTÃO (OBS é NULA)

(24) SE (PA é Baixa) E (DIV é Poucas) E (JAN é Alta) E (DIS é Baixa) E (TAM é Pequeno) E (AM é Alto) ENTÃO (OBS é NULA)

(25) SE (PA é Média) E (DIV é Poucas) E (JAN é Alta) E (DIS é Baixa) E (TAM é Pequeno) E (AM é Alto) ENTÃO (OBS é NULA)

(26) SE (PA é Baixa) E (DIV é Poucas) E (JAN é Média) E (DIS é Baixa) E (TAM é Pequeno) E (AM é Médio) ENTÃO (OBS é NULA)

(27) SE (PA é Média) E (DIV é Muitas) E (JAN é Média) E (DIS é Baixa) E (TAM é Pequeno) E (AM é Médio) ENTÃO (OBS é NULA)

(28) SE (PA é Baixa) E (DIV é Muitas) E (JAN é Alta) E (DIS é Baixa) E (TAM é Pequeno) E (AM é Médio) ENTÃO (OBS é NULA)

(29) SE (PA é Média) E (DIV é Muitas) E (JAN é Alta) E (DIS é Baixa) E (TAM é Pequeno) E (AM é Médio) ENTÃO (OBS é NULA)

(30) SE (PA é Baixa) E (DIV é Muitas) E (JAN é Média) E (DIS é Baixa) E (TAM é Pequeno) E (AM é Alto) ENTÃO (OBS é NULA)

(31) SE (PA é Média) E (DIV é Muitas) E (JAN é Média) E (DIS é Baixa) E (TAM é Pequeno) E (AM é Alto) ENTÃO (OBS é NULA)

(32) SE (PA é Baixa) E (DIV é Muitas) E (JAN é Alta) E (DIS é Baixa) E (TAM é Pequeno) E (AM é Alto) ENTÃo (OBS é NULA)

(33) SE (PA é Média) E (DIV é Muitas) E (JAN é 
Alta) E (DIS é Baixa) E (TAM é Pequeno) E (AM é Alto) ENTÃO (OBS é NULA)

(34) SE (PA é Média) E (DIV é Poucas) E (JAN é Alta) E (DIS é Média) E (TAM é Pequeno) E (AM é Alto) ENTÃO (OBS é ALTA)

(35) SE (PA é Média) E (DIV é Muitas) E (JAN é Alta) E (DIS é Média) E (TAM é Pequeno) E (AM é Alto) ENTÃO (OBS é ALTA)

(36) SE (PA é Média) E (DIV é Muitas) E (JAN é Alta) E (DIS é Alta) E (TAM é Pequeno) E (AM é Alto) ENTÃO (OBS é BAIXA)

(37) SE (PA é Média) E (DIV é Poucas) E (JAN é Alta) E (DIS é Alta) E (TAM é Pequeno) E (AM é Alto) ENTÃO (OBS é MUITO BAIXA)

(38) SE (PA é Alta) E (DIV é Muitas) E (JAN é Alta) E (DIS é Alta) E (TAM é Pequeno) E (AM é Alto) ENTÃO (OBS é MUITO BAIXA)

(39) SE (PA é Baixa) E (DIV é Poucas) E (JAN é Baixa) E (DIS é Baixa) E (TAM é Pequeno) E (AM é Baixo) ENTÃO (OBS é MUITO BAIXA)

(40) SE (PA é Média) E (DIV é Poucas) E (JAN é Baixa) E (DIS é Baixa) E (TAM é Pequeno) E (AM é Baixo) ENTÃO (OBS é MUITO BAIXA)

(41) SE (PA é Alta) E (DIV é Muitas) E (JAN é Baixa) E (DIS é Alta) E (TAM é Pequeno) E (AM é Alto) ENTÃO (OBS é MUITO BAIXA)

(42) SE (PA é Baixa) E (DIV é Poucas) E (JAN é Baixa) E (DIS é Baixa) E (TAM é Pequeno) E (AM é Baixo) ENTÃO (OBS é MUITO BAIXA)

(43) SE (PA é Média) E (DIV é Poucas) E (JAN é Baixa) E (DIS é Baixa) E (TAM é Pequeno) E (AM é Baixo) ENTÃO (OBS é MUITO BAIXA)

(44) SE (PA é Alta) E (DIV é Muitas) E (JAN é Baixa) E (DIS é Alta) E (TAM é Pequeno) E (AM é Alto) ENTÃO (OBS é MUITO BAIXA)

(45) SE (PA é Média) E (DIV é Muitas E (JAN é Baixa) E (DIS é Baixa) E (TAM é Pequeno) E (AM é Baixo) ENTÃO (OBS é MUITO BAIXA)

(46) SE (PA é Alta) E (DIV é Muitas) E (JAN é Baixa) E (DIS é Média E (TAM é Pequeno) E (AM é Alto) ENTÃO (OBS é MUITO BAIXA)

(47) SE (PA é Baixa) E (DIV é Poucas) E (JAN é Baixa) E (DIS é Média) E (TAM é Pequeno) E (AM é Baixo) ENTÃO (OBS é MUITO BAIXA)

(48) SE (PA é Média) E (DIV é Muitas) E (JAN é Baixa) E (DIS é Média) E (TAM é Pequeno) E (AM é Baixo) ENTÃO (OBS é MUITO BAIXA)
(49) SE (PA é Alta) E (DIV é Poucas) E (JAN é Baixa) E (DIS é Alta ) E (TAM é Pequeno) E (AM é Alto) ENTÃO (OBS é MUITO BAIXA)

(50) SE (PA é Alta) E (DIV é Poucas) E (JAN é Baixa) E (DIS é Baixa) E (TAM é Pequeno) E (AM é Alto) ENTÃO (OBS é MUITO BAIXA)

(51) SE (PA é Baixa) E (DIV é Muitas) E (JAN é Baixa) E (DIS é Baixa) E (TAM é Pequeno) E (AM é Média) ENTÃO (OBS é MEDIA)

(52) SE (PA é Baixo) E (DIV é Pouca) E (JAN é Baixa) E (DIS é Baixa) E (TAM é Pequeno) E (AM é Médio) ENTÃO (OBS é MEDIA)

(53) SE (PA é Médio) E (DIV é Pouca) E (JAN é Baixa) E (DIS é Baixa) E (TAM é Pequeno) E (AM é Médio) ENTÃO (OBS é MEDIA)

(54) SE (PA é Baixa) E (DIV é Muitas) E (JAN é Baixa) E (DIS é Baixa) E (TAM é Grande) E (AM é Média) ENTÃO (OBS é MEDIA)

(55) SE (PA é Média) E (DIV é Muitas) E (JAN é Baixa) E (DIS é Baixa) E (TAM é Grande) E (AM é Média) ENTÃO (OBS é MEDIA)

(56) SE (PA é Baixo) E (DIV é Pouca) E (JAN é Baixa) E (DIS é Baixa) E (TAM é Grande) E (AM é Média) ENTÃO (OBS é MEDIA)

(57) SE (PA é Médio) E (DIV é Pouca) E (JAN é Baixa) E (DIS é Baixa) E (TAM é Grande) E (AM é Média) ENTÃO (OBS é MEDIA)

(58) SE (PA é Baixa) E (DIV é Poucas) E (JAN é Baixa) E (DIS é Baixa) E (TAM é Pequeno) E (AM é Médio) ENTÃO (OBS é MEDIA)

(59) SE (PA é Média) E (DIV é Poucas) E (JAN é Baixa) E (DIS é Baixa) E (TAM é Pequeno) E (AM é Médio) ENTÃO (OBS é MEDIA)

(60) SE (PA é Baixa) E (DIV é Pouca) E (JAN é Baixa) E (DIS é Baixa) E (TAM é Pequeno) E (AM é Média) ENTÃO (OBS é MEDIA)

(61) SE (PA é Média) E (DIV é Poucas) E (JAN é Baixa) E (DIS é Baixa) E (TAM é Pequeno) E (AM é Médio) ENTÃO (OBS é MEDIA)

(62) SE (PA é Baixo) E (DIV é Pouca) E (JAN é Baixa) E (DIS é Alta ) E (TAM é Pequeno) E (AM é Médio) ENTÃO (OBS é MEDIA)

(63) SE (PA é Médio) E (DIV é Pouca) E (JAN é Baixa) E (DIS é Alta) E (TAM é Pequeno) E (AM é Médio) ENTÃO (OBS é MEDIA)

(64) SE (PA é Baixa) E (DIV é Muitas) E (JAN é Baixa) E (DIS é Alta) E (TAM é Grande) E (AM é 
Média) ENTÃO (OBS é MEDIA)

(65) SE (PA é Média) E (DIV é Muitas) E (JAN é Baixa) E (DIS é Alta) E (TAM é Grande) E (AM é Média) ENTÃO (OBS é MEDIA)

(66) SE (PA é Baixa) E (DIV é Poucas) E (JAN é Baixa) E (DIS é Baixa) E (TAM é Pequeno) E (AM é Médio) ENTÃO (OBS é MEDIA)

(67) SE (PA é Média) E (DIV é Muitas) E (JAN é Baixa) E (DIS é Baixa) E (TAM é Pequeno) E (AM é Médio) ENTÃO (OBS é MEDIA)

(68) SE (PA é Baixa) E (DIV é Muitas) E (JAN é Baixa) E (DIS é Baixa) E (TAM é Pequeno) E (AM é Médio) ENTÃO (OBS é MEDIA)

(69) SE (PA é Média) E (DIV é Muitas) E (JAN é Baixa) E (DIS é Baixa) E (TAM é Pequeno) E (AM é Médio) ENTÃO (OBS é MEDIA)

(70) SE (PA é Baixa) E (DIV é Poucas) E (JAN é Baixa) E (DIS é Baixa) E (TAM é Pequeno) E (AM é Médio) ENTÃO (OBS é MEDIA)

(71) SE (PA é Baixa) E (DIV é Poucas) E (JAN é Baixa) E (DIS é Média) E (TAM é Pequeno) E (AM é Médio) ENTÃO (OBS é MEDIA)

(72) SE (PA é Baixa) E (DIV é Poucas) E (JAN é Baixa) E (DIS é Média) E (TAM é Pequeno) E (AM é Médio) ENTÃO (OBS é MEDIA)

(73) SE (PA é Médio) E (DIV é Pouca) E (JAN é Média) E (DIS é Média) E (TAM é Pequeno) E (AM é Médio) ENTÃO (OBS é ALTA)

(74) SE (PA é Baixo) E (DIV é Pouca) E (JAN é Média) E (DIS é Média) E (TAM é Pequeno) E (AM é Alto) ENTÃO (OBS é ALTA)

(75) SE (PA é Médio) E (DIV é Pouca) E (JAN é Média) E (DIS é Média) E (TAM é Pequeno) E (AM é Alto) ENTÃO (OBS é MEDIA)

(76) SE (PA é Baixo) E (DIV é Pouca) E (JAN é Média) E (DIS é Média) E (TAM é Grande) E (AM é Média) ENTÃO (OBS é ALTA)

(77) SE (PA é Médio) E (DIV é Pouca) E (JAN é Média) E (DIS é Média) E (TAM é Grande) E (AM é Média) ENTÃO (OBS é ALTA)

(78) SE (PA é Baixo) E (DIV é Pouca) E (JAN é Média) E (DIS é Média) E (TAM é Grande) E (AM é Alto) ENTÃO (OBS é MEDIA)

(79) SE (PA é Média) E (DIV é Pouca) E (JAN é Média) E (DIS é Média) E (TAM é Grande) E (AM é Alto) ENTÃO (OBS é MEDIA)

(80) SE (PA é Baixa) E (DIV é Pouca) E (JAN é
Média) E (DIS é Média) E (TAM é Pequeno) E (AM é Média) ENTÃO (OBS é NULA)

(81) SE (PA é Baixa) E (DIV é Muitas) E (JAN é Média) E (DIS é Média) E (TAM é Pequeno) E (AM é Média) ENTÃO (OBS é NULA)

(82) SE (PA é Baixa) E (DIV é Muitas) E (JAN é Média) E (DIS é Média) E (TAM é Pequeno) E (AM é Alto) ENTÃO (OBS é BAIXA)

(83) SE (PA é Média) E (DIV é Muitas) E (JAN é Média) E (DIS é Média) E (TAM é Pequeno) E (AM é Alto) ENTÃO (OBS é BAIXA)

(84) SE (PA é Baixa) E (DIV é Muitas) E (JAN é Média) E (DIS é Média) E (TAM é Grande) E (AM é Média) ENTÃO (OBS é BAIXA)

(85) SE (PA é Média) E (DIV é Muitas) E (JAN é Média) E (DIS é Média) E (TAM é Grande) E (AM é Média) ENTÃO (OBS é BAIXA)

(86) SE (PA é Baixa) E (DIV é Muitas) E (JAN é Média) E (DIS é Média) E (TAM é Grande) E (AM é Alto) ENTÃO (OBS é BAIXA)

(87) SE (PA é Média) E (DIV é Muitas) E (JAN é Média) E (DIS é Média) E (TAM é Grande) E (AM é Alto) ENTÃO (OBS é BAIXA)

(88) SE (PA é Baixa) E (DIV é Poucas) E (JAN é Média) E (DIS é Média) E (TAM é Pequeno) E (AM é Médio) ENTÃO (OBS é MUITO BAIXA)

(89) SE (PA é Média) E (DIV é Poucas) E (JAN é Média) E (DIS é Média) E (TAM é Pequeno) E (AM é Médio) ENTÃO (OBS é MUITO BAIXA)

(90) SE (PA é Baixa) E (DIV é Poucas) E (JAN é Alta) E (DIS é Média) E (TAM é Pequeno) E (AM é Médio) ENTÃO (OBS é MUITO BAIXA)

(91) SE (PA é Média) E (DIV é Poucas) E (JAN é Alta) E (DIS é Média) E (TAM é Pequeno) E (AM é Médio) ENTÃO (OBS é MUITO BAIXA)

(92) SE (PA é Baixa) E (DIV é Poucas) E (JAN é Média) E (DIS é Média) E (TAM é Pequeno) E (AM é Alto) ENTÃO (OBS é ALTA)

(93) SE (PA é Média) E (DIV é Muitas) E (JAN é Média) E (DIS é Média) E (TAM é Pequeno) E (AM é Alto) ENTÃO (OBS é MEDIA)

(94) SE (PA é Baixa) E (DIV é Poucas) E (JAN é Alta) E (DIS é Média) E (TAM é Pequeno) E (AM é Alto) ENTÃO (OBS é BAIXA)

(95) SE (PA é Média) E (DIV é Poucas) E (JAN é Alta) E (DIS é Média) E (TAM é Pequeno) E (AM é Alto) ENTÃO (OBS é BAIXA) 
(96) SE (PA é Baixa) E (DIV é Poucas) E (JAN é Média) E (DIS é Média) E (TAM é Pequeno) E (AM é Médio) ENTÃO (OBS é BAIXA)

(97) SE (PA é Média) E (DIV é Muitas) E (JAN é Média) E (DIS é Média) E (TAM é Pequeno) E (AM é Médio) ENTÃO (OBS é MEDIA)

(98) SE (PA é Baixa) E (DIV é Muitas) E (JAN é Alta) E (DIS é Média) E (TAM é Pequeno) E (AM é Médio) ENTÃO (OBS é MEDIA)

(99) SE (PA é Média) E (DIV é Muitas) E (JAN é Alta) E (DIS é Média) E (TAM é Pequeno) E (AM é Médio) ENTÃO (OBS é MEDIA)

(100) SE (PA é Baixa) E (DIV é Muitas) E (JAN é Média) E (DIS é Média) E (TAM é Pequeno) E (AM é Alto) ENTÃO (OBS é MEDIA)

(101) SE (PA é Média) E (DIV é Muitas) E (JAN é Média) E (DIS é Média) E (TAM é Pequeno) E (AM é Alto) ENTÃO (OBS é MEDIA)

(102) SE (PA é Baixa) E (DIV é Muitas) E (JAN é Alta) E (DIS é Média) E (TAM é Pequeno) E (AM é Alto) ENTÃO (OBS é MEDIA)

(103) SE (PA é Média) E (DIV é Muitas) E (JAN é Alta) E (DIS é Média) E (TAM é Pequeno) E (AM é Alto) ENTÃO (OBS é MEDIA)

Recebido em 2 Fevereiro 2011- Received on February 2, 2011.

Aceito em 7 Março, 2011 - Accepted on March 3, 2011. 